\title{
The effects of nursing of Roy adaptation model on the elderly hypertensive: a randomised control study
}

\author{
Jiali Zhang" ${ }^{1 \#}$ Lilan Guo ${ }^{2 \#}$,Jundan $\mathrm{Mao}^{2}, \mathrm{Xue}_{\mathrm{Qi}}{ }^{2}$, Liqun Chen ${ }^{2}$, Hui Huang ${ }^{2}$, Yanling Sun ${ }^{2}$, Xiaoyue Yang \\ ${ }^{1}$ Department of Medicine, the Fifth Affiliated Hospital of Sun Yat-sen University, Zhuhai, China; ${ }^{2}$ Geriatrics Department, the Fifth Affiliated \\ Hospital of Sun Yat-sen University, Zhuhai, China; ${ }^{3}$ Nursing Department, the Fifth Affiliated Hospital of Sun Yat-sen University, Zhuhai, China \\ Contributions: (I) Conception and design: J Zhang, L Guo, X Yang; (II) Administrative support: X Yang; (III) Provision of study materials or patients: \\ J Mao, X Qi; (IV) Collection and assembly of data: J Zhang, L Guo, H Huang, Y Sun; (V) Data analysis and interpretation: All authors; (VI) \\ Manuscript writing: All authors (VII) Final approval of manuscript: All authors. \\ \#These authors contributed equally to this work. \\ Correspondence to: Xiaoyue Yang. Nursing Department, the Fifth Affiliated Hospital of Sun Yat-sen University, No. 58, Meihua East Road, Xiangzhou \\ District, Zhuhai 519000, China. Email: zdwylnk2021@126.com.
}

Background Hypertension is a chronic disease affecting middle-aged and elderly patients worldwide. Self-management behavior plays a critical role in maintaining blood pressure. Roy adaptation model (RAM), which was proposed by Roy in 1970, has been used to guide hypertension nursing to improve selfmanagement behavior of elderly patients. In the study, to explore the effect of nursing based on the RAM on the self-management behavior of elderly hypertensive patients.

Methods: A total of 120 elderly hypertensive patients admitted from June 2020 to March 2021 were selected and randomly divided into a control group and observation group based on odd and even numbers of the admission order, with 60 cases in each group. The control group received routine nursing measures while the observation group was given nursing based on the RAM. The self-management behavior, medication compliance, quality of life, and blood pressure control effect were compared between the two groups.

Results: The scores of the self-efficacy and self-management behaviors of the observation group and the total score of the scale were higher than those of the control group $(\mathrm{P}<0.05)$; the observation group patients' medication compliance score was $6.57 \pm 1.47$, which was higher than that of the control group $(4.90 \pm 2.16)$ $(\mathrm{P}<0.01)$; physiological function, physical pain, energy, social function, emotional function, mental health scores, and total scores of the SF-36 scale in the observation group were all higher than those of the control group $(\mathrm{P}<0.05)$, while comparison of the general health status dimension scores showed no statistically significant difference; the systolic blood pressure and diastolic blood pressure of the observation group were respectively lower than those of the control group $(\mathrm{P}<0.05)$; The blood pressure control compliance rate of the observation group was $85.4 \%$, which was higher than that of the control group $64.6 \%(\mathrm{P}<0.05)$.

Conclusions: Nursing intervention based on Roy's adaptation model can better enhance the self-efficacy and self-management ability of elderly hypertensive patients, have a positive effect on promoting healthy behavior changes and improving the quality of life, improve medication compliance, and achieve better blood pressure control effects.

Trial Registration: Chinese Clinical Trial Registry ChiCTR2100052466.

Keywords: Roy adaptation model (RAM); hypertension; self-management behavior; medication compliance; quality of life

Submitted Sep 14, 2021. Accepted for publication Nov 09, 2021.

doi: 10.21037/apm-21-2803

View this article at: https://dx.doi.org/10.21037/apm-21-2803 


\section{Introduction}

Hypertension is a common disease worldwide, with high prevalence, high complications, a high disability rate, and a low control rate (1). The disease is potentially accompanied with cerebrovascular diseases such as stroke and coronary heart disease, and is the main cause of premature death worldwide (2). Recently, epidemiological data suggest that the elderly have high systolic blood pressure and low diastolic blood pressure. The higher the systolic blood pressure, the more cardiovascular and cerebrovascular complications, the higher the mortality rate, especially stroke. In addition, the blood pressure fluctuations are greatly due to the hardening of blood vessels in elderly patients. According to estimates by the World Health Organization, 1.13 billion people worldwide were suffering from hypertension in 2019, representing a significant increase from the 5.94 billion in 1975 (2). In recent years, the prevalence of hypertension has shown a rapid growth trend and is highest in adults and the elderly, in whom it whom from $30.0 \%$ to $71.6 \%$ (3). Hypertension is a chronic disease that requires long-term management, and self-management behavior is the key to its control. The 2020 International Society of Hypertension global hypertension practice guidelines (4) propose that to stabilize blood pressure and reduce damage to target organs and other complications, all patients with hypertension should participate in self-management to varying degrees, including adopting a healthy lifestyle, maintaining emotional stability, regularly monitoring blood pressure, and taking standardized medication. However, because elderly hypertensive patients are often memory impaired, have poor vision and decreased self-confidence, and may suffer from anxiety, depression, or loneliness they may fail to take medicine as prescribed, and are prone to poor selfmanagement, resulting in poor blood pressure control, and a decline in their quality of life. Therefore, helping this group enhance their confidence and improve self-management and medication compliance is of profound significance. The Roy adaptation model (RAM) was first proposed by the American nursing scientist Roy in 1970 (5), and has been used to guide interdisciplinary education, knowledge development, practice, and research in nursing (6). de Oliveira TC demonstrated that six diagnoses include restricted mobility, gait, and/or coordination; impairment of a primary sense; sleep deprivation; low self-esteem; role transition and loneliness after behavior and stimulus evaluation. The results suggested that the nursing goals and RAM interventions were important. Once that allowed in a general way, the ineffective responses substitution for adaptive ones (7).

Roy adaptation model was first proposed by Callista Roy who believes that humans are a whole adaptive system and life is a process of continuous adaptation to various stimuli of internal and external environment. The purpose of nursing is to promote people to constantly adapt to the changes of the environment and maintain human's physical, psychological and social integrity for improving the fitness level of the nation's population. Based on the concept of holism, the RAM discusses the overall adaptation process, adaptation mode, and adaptation mechanism of humans as a system with psychological, physiological, and social adaptation to various internal and external environmental stimuli. To improve the adaptive response of patients, it is necessary to properly intervene in this process and the RAM can guide medical staff to judge and evaluate the stimulus that leads to a patients adaptation problems, so as to implement comprehensive and targeted nursing interventions, reduce or avoid ineffective responses, expand the scope of the adaptive response, and improve the patient's health level (8). This study aims to discuss the effects of the RAM applied to hypertension nursing on the self-management behavior of elderly patients, their medication compliance, quality of life, and blood pressure control effect, hoping to provide a reference for clinical work. We present the following article in accordance with the CONSORT reporting checklist (available at https:// dx.doi.org/10.21037/apm-21-2803).

\section{Methods}

\section{Objects}

A total of 120 elderly hypertensive patients admitted to our hospital from June 2020 to March 2021 were selected using a convenient sampling method then divided into a control group and observation group based on odd and even numbers of their admission order, with 60 patients in each group and allocation ratio is $1: 1$. Inclusion criteria: (I) meeting the diagnostic criteria of hypertension (9); (II) age $\geq 65$ years of age; (III) taking at least one hypertensive drug; (IV) clear consciousness and stable condition; (V) patient and family members were informed and agreed to participate in the study. Exclusion criteria: (I) elderly patients with secondary hypertension; (II) people unable to take care of themselves; (III) disease such as major mental illness, cognitive impairment, senile dementia, or malignant tumors; (IV) people with writing or verbal communication disabilities. In the control group, there were 28 males and 
32 females, aged $65-85$ (average $75.27 \pm 8.12$ years), of whom 37 had junior high school education and below and 23 had high school education and above. In the observation group, there were 29 males and 31 females, aged 65 to 85 years (average $74.22 \pm 7.97$ years), of whom 34 cases had junior high school education and below and 26 cases were high school education and above. Comparison of the general information of the two groups showed that the difference was not statistically significant $(\mathrm{P}>0.05)$ but was comparable. All procedures performed in this study involving human participants were in accordance with the Declaration of Helsinki (as revised in 2013). The study was approved by Ethics Committee of the Fifth Affiliated Hospital of Sun Yat-sen University (No. k242-1) and informed consent was taken from all the patients.

\section{Study methods}

\section{Intervention method}

A health file was established for each participant. Patients in the control group were given routine nursing in accordance with the routine care of those with elderly hypertensive disease, such as blood pressure monitoring, reducing blood pressure over time, and good health education. In the experimental group, RAM nursing procedures were used throughout the entire nursing process on the basis of routine care, and corresponding nursing plans were formulated according to the patient's condition as follows: A continuous intervention mode was provided during hospitalization and follow up once per month after discharge, a total of three times: (I) primary assessment: intervention team members collected basic and clinical data of patients through observation, communication, and physical function examination to evaluate factors including the their mutual dependence, physiological function, role function, and self-concept, and distinguish between adaptive and ineffective responses. (II) Secondary assessment: this involved collecting and identifying the inherent stimuli, related stimuli, and main stimuli that caused the patient to have an ineffective response to further clarify the influencing factors. (III) Nursing diagnosis: this involved proposing the patient's existing or potential nursing diagnosis based on the above data collected and summarized as well as the evidence collected. (IV) Goal formulating: this involved formulating corresponding nursing goals, actively transforming ineffective responses into adaptive responses, and expanding the scope of adaptability to achieve satisfactory clinical nursing effects. (V) Nursing measures: this involved formulating relevant nursing measures for various adaptability problems to reduce the frequency and intensity of corresponding stimulation and promote the adaptability of patients in the following four aspects: Physiological function, interdependence, role function, and self-concept. (i) Physiological function: when elderly hypertensive patients were not comfortable because of dizziness, headaches, or lack of physical strength, they were provided with a quiet and comfortable environment, and asked to rest in bed. The reasons why hypertension causes discomfort were explained, and the patient was then introduced to and guided through relaxation techniques and coping methods during attacks. The blood pressure of elderly patients is easily affected by body position, meals, daytime changes, mood, seasons, and climate, and the circadian rhythm of blood pressure is mostly double-peak and double-valley curve. Blood pressure measurement time and procedure could then be then standardized to address these fluctuations. The daily energy requirements of patients were also calculated, and appropriate dietary intervention to control caloric intake, sodium, and salt intake, and to increase the intake of calcium and potassium was implemented. Any difficulties with sleep and bowel habits were also determined and promptly treated. Based on the patient's condition and individual body tolerance, individualized exercise guidance was formulated to help develop good living habits. (ii) Interdependence: the effective encouragement and supervision of family members will increase the confidence of patients in long-term treatment and overcoming the disease, so family members were encouraged to maintain a close relationship with patients, to make them feel respected, and cultivate hobbies and other ways of connecting. (iii) Role function: patients were gradually made aware of their own importance to the whole family and their community, to enhance their sense of responsibility. In addition, patients were encouraged to positively face the disease and participate in the formulation of treatment and nursing plans and actively cooperate with staff. (iv) Self-concept: nursing staff actively communicated with patients to keep abreast of their mental state. Diversified health education, such as health manuals, videos playing in the ward, WeChat posts, and health department lectures were used to facilitate their understanding and assessment of their own diseases, correctly and actively control their disease, and maintain a good attitude towards it. For patients with negative self-concepts, nursing staff established groups where patients were exposed to others who were successfully coping with the disease or had more 


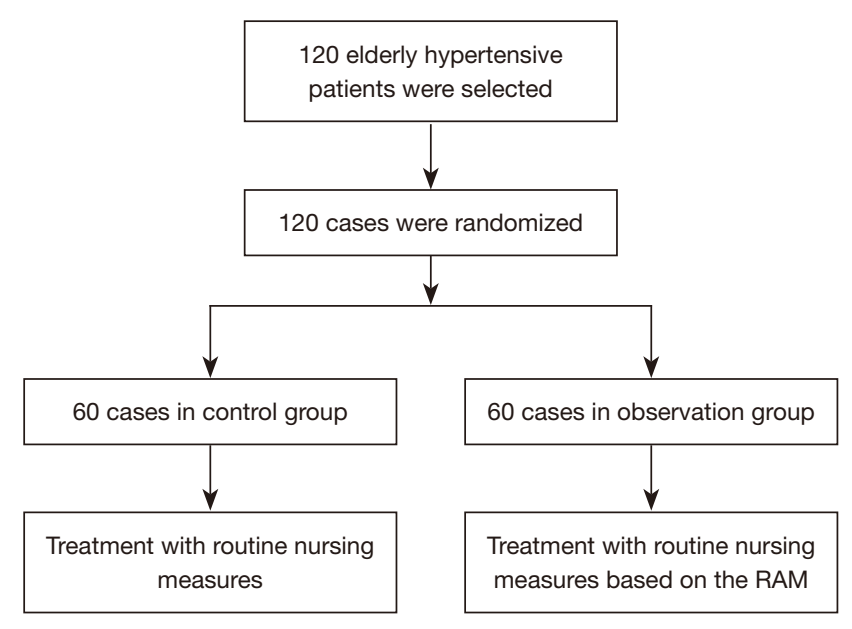

Figure 1 A flow diagram concerning participant enrollment. RAM, roy adaptation model.

serious diseases, to maintain their inner balance and form a positive ego ideal, and to reduce the gap between the expectations of the image and behavior and the reality. In addition, patients were encouraged to use the successful experience of coping with difficulties in the past to formulate strategies and methods for coping with illness, so that they could maintain self-consistency stability.

\section{Observation indexes}

(I) Self-management behavior. A hypertension patient's selfmanagement behavior evaluation table prepared by Yang et al. (10) was used to assess patients' self-management behavior level, including contents in drug treatment, daily life, treatment compliance, and health behavior. The scale consists of the following five levels: completely unconfident, basically unconfident, uncertain, basically confident, and completely confident, which were assigned a score of $0-4$, and positively correlated with patients' self-management behavior. The Cronbach's $\alpha$ of the scale is 0.804 , while a self-management behavior score $\geq 31$ is defined as a high level, $23-30$ is a medium level, and $\leq 22$ is a low level. (II) Self-management behavior. This scale was prepared by Zhao et al. (11) and has a total Cronbach's $\alpha$ of 0.914 and validity of 0.910 . It includes six dimensions including disease monitoring, diet management, work and rest management, exercise management, emotional management, and medication management, with a total of 33 items. The Likert 5 -level scoring method is used as follows: $1=$ never, $2=$ rarely, $3=$ sometimes, $4=$ often, $5=$ always. The total score of the scale is $33-165$ points, and the higher the score, the better the patient's self-management behavior. The self-management level was divided into three levels: low ( $<60$ points), medium ( $60-80$ points), and high ( $>80$ points). (III) To evaluate medication compliance. Morishy's medication compliance scale (MMAS-8) (12) was used, which has a Cronbach's $\alpha$ of 0.83 . There are eight items in the scale, and the options for items 1 to 7 are "Yes" (counting 0 point) and "No" (counting 1 point), but the scoring of the fifth item is the opposite. The options of the eighth item are "never", "barely", "sometimes", "often", and "always", which are respectively allocated $1,0.75,0.5,0.25$, and 0 points. The total score is 8 points, and below 6 points means low compliance, 6 to 7 points means medium compliance, and 8 points means high compliance. (IV) Quality of life. The SF-36 Quality of life evaluation scale developed by the Boston Institute of Health was used to evaluate eight dimensions: physiological function, physiological competency, physical pain, energy, social function, emotional function, mental health, and general health status. Practice has proved that the scale has good reliability and validity (13). The total score for each dimension is 100 points, and the higher the score, the better the expression of the dimension. (V) Blood pressure control. A unified sphygmomanometer was used to measure blood pressure, and from the start of the intervention to 6 months after the intervention, those with recordings below $140 / 90 \mathrm{mmHg}$ were regarded as reaching the blood pressure standard. The blood pressure compliance rate $=$ the number of patients reaching the blood pressure standard/total number of patients $\times 100 \%$.

\section{Statistical methods}

SPSS20.0 statistical software was used for data analysis. Measurement data conforming to the normal distribution were expressed as mean \pm standard deviation $(\bar{x} \pm \mathrm{s})$, and $t$-test was performed for comparison between groups. The count data were expressed by the number of cases and percentage (\%), and the comparison between groups was performed by the $\chi^{2}$ test. The inspection level $\alpha=0.05$, and $\mathrm{P}<0.05$ indicates that the difference was statistically significant. All the observation indexes were primary endpoints.

\section{Results}

Comparison of self-efficacy and self-management behavior between two groups

A flow diagram concerning participant enrollment has 
Table 1 Comparison of baseline data between the two groups

\begin{tabular}{|c|c|c|c|c|}
\hline Variable & Observation group & Control group & $\chi^{2}$ & $P$ \\
\hline Male & $33(55.0)$ & $32(53.3)$ & & \\
\hline Female & $27(45.0)$ & $28(46.7)$ & & \\
\hline Age (years), n (\%) & & & 5.209 & 0.074 \\
\hline $70-80$ & $32(53.3)$ & $20(33.3)$ & & \\
\hline Above 80 & $11(18.3)$ & $13(21.7)$ & & \\
\hline
\end{tabular}

Table 2 Comparison of self-efficacy between the two groups after intervention (points, $\bar{x}_{ \pm \mathrm{s}} \mathrm{n}=60$ )

\begin{tabular}{lcccc}
\hline Self-efficacy & Observation group & Control group & $t$ & $P$ \\
\hline Daily life & $11.04 \pm 1.09$ & $10.31 \pm 1.99$ & 2.225 & 0.029 \\
Healthy behavior & $7.69 \pm 0.83$ & $7.21 \pm 1.40$ & 2.042 & 0.045 \\
Medical treatment & $11.58 \pm 0.99$ & $11.06 \pm 1.36$ & 2.149 & 0.034 \\
Treatment compliance & $10.65 \pm 1.47$ & $8.33 \pm 2.48$ & 5.564 & 0 \\
Total score & $40.96 \pm 3.72$ & $36.92 \pm 4.88$ & 4.563 & 0 \\
\hline
\end{tabular}

Table 3 Comparison of self-management behavior between the two groups after intervention (points, $\bar{x}_{ \pm s,} \mathrm{n}=60$ )

\begin{tabular}{|c|c|c|c|c|}
\hline Self-management & Observation group & Control group & $t$ & $\mathrm{P}$ \\
\hline Illness monitoring & $15.63 \pm 2.69$ & $11.63 \pm 4.02$ & 5.722 & 0 \\
\hline Diet management & $44.94 \pm 7.67$ & $36.63 \pm 6.41$ & 5.761 & 0 \\
\hline Exercise management & $12.46 \pm 3.11$ & $11.90 \pm 2.91$ & 0.914 & 0.363 \\
\hline Emotion management & $31.48 \pm 3.78$ & $26.38 \pm 6.63$ & 4.634 & 0 \\
\hline Total score & $148.02 \pm 11.44$ & $124.04 \pm 15.04$ & 8.792 & 0.0 \\
\hline
\end{tabular}

been depicted as in Figure 1. The data was collected from June 2020 to March 2021. The comparison of baseline data, self-efficacy and self-management behavior between the two groups of patients after intervention is shown in Tables 1-3. The results showed that there are no statistical differences between the two groups in gender ratios and age. The scores of four dimensions of self-efficacy, scores of six dimensions of self-management behavior, and the total score of the scale in the observation group were significantly higher than in the control group, and the difference was statistically significant $(\mathrm{P}<0.05)$.

\section{Comparison of medication compliance between the two groups}

Comparison of medication compliance between the two groups after intervention is shown in Table 4 and shows the medication compliance score in the observation group was significantly higher than that of the control group, and the difference was statistically significant $(\mathrm{P}<0.01)$. 
Table 4 Comparison of medication compliance between the two groups after intervention (points, $\bar{x}_{ \pm \mathrm{s}}, \mathrm{n}=60$ )

\begin{tabular}{lcccc}
\hline & Observation group & Control group & $t$ & $\mathrm{P}$ \\
\hline Medication compliance & $6.57 \pm 1.47$ & $4.90 \pm 2.16$ & 4.427 & 0.0 \\
\hline
\end{tabular}

Table 5 Comparison of quality of life between the two groups after intervention (points, $\bar{x} \pm \mathrm{s}, \mathrm{n}=60$ )

\begin{tabular}{|c|c|c|c|c|}
\hline Quality of life & Observation group & Control group & $t$ & $\mathrm{P}$ \\
\hline Physiological competency & $78.13 \pm 30.35$ & $49.38 \pm 36.36$ & 4.206 & 0 \\
\hline Physical pain & $86.96 \pm 14.03$ & $77.33 \pm 22.44$ & 2.52 & 0.013 \\
\hline General health status & $66.21 \pm 19.08$ & $59.10 \pm 19.88$ & 1.786 & 0.077 \\
\hline Social function & $89.32 \pm 16.10$ & $81.78 \pm 19.28$ & 2.08 & 0.04 \\
\hline Emotional function & $93.06 \pm 19.40$ & $79.17 \pm 37.43$ & 2.283 & 0.025 \\
\hline Mental health & $74.42 \pm 6.89$ & $66.08 \pm 11.58$ & 4.284 & 0 \\
\hline Total score & $642.46 \pm 75.13$ & $553.05 \pm 111.20$ & 4.616 & 0.0 \\
\hline
\end{tabular}

Table 6 Comparison of blood pressure control effect between the two groups after intervention $\left(\bar{x}_{ \pm \mathrm{S}}, \mathrm{n}=60\right)$

\begin{tabular}{lcccc}
\hline Blood pressure & Observation group & Control group & $t / \chi^{2}$ & $P$ \\
\hline Systolic blood pressure $(\mathrm{mmHg})$ & $135.77 \pm 7.56$ & $142.50 \pm 11.06$ & -3.480 & 0.001 \\
Diastolic blood pressure $(\mathrm{mmHg})$ & $73.81 \pm 6.50$ & $77.65 \pm 8.81$ & -2.427 & 0.017 \\
Standard blood pressure, $\mathrm{n}(\%)$ & $41(85.4)$ & $31(64.6)$ & 5.556 & 0.018 \\
\hline
\end{tabular}

\section{Comparison of the quality of life between the two groups}

Comparison of the quality of life between the two groups after intervention is shown in Table 5. The scores of seven dimensions (physiological function, physiological competency, physical pain, energy, social function, emotional function, and mental health) and total score of patients in the observation group were higher than those in the control group, and the difference was statistically significant $(\mathrm{P}<0.05)$. The comparison of scores in the general health status dimension, showed no statistically significant difference $(\mathrm{P}>0.05)$.

\section{Comparison of blood pressure control effect between the two groups}

Comparison of blood pressure control effect between the two groups after intervention is shown Table 6. Both the diastolic blood pressure and systolic blood pressure in the observation group were lower than that in the control group. The blood pressure compliance rate in the observation group was higher than that in the control group, and the difference was statistically significant $(\mathrm{P}<0.05)$.

\section{Discussion}

Hypertension is a common chronic disease seen in clinical practice and features a long-term course, high morbidity, and difficulty to quickly cure. The onset of hypertension is closely related to heredity and lifestyle factors, and the disease has a significant impact on health, not only damaging major organs including the heart, blood vessels of the brain, and kidney, but also interfering with the mental health of patients. Hypertension also imposes a significant economic burden on families, communities, and society at large (14). Hypertension in the elderly not only affects the normal life of patients seriously, but also brings pain to patients. 
Therefore, active prevention is needed. In the prevention of hypertension, the elderly demand getting enough sleep, eating balanced meals, appropriate exercise and maintaining a good state of mind. The China Cardiovascular Disease Report 2018 showed that there were approximately 245 million hypertension patients in China, and in many regions, hypertension-related cardiovascular diseases surpassed tumors, becoming the main cause of mortality and a major public health problem (15). Hypertensive disease management emphasizes prevention more than cure, and all hypertension patients should participate in their health management. In recent years, while prevention and management measures have been continuously implemented, the disease cognition, self-management ability, adherence to medication compliance, and blood pressure control effect of patients are still poor, especially in elderly patients. Good self-management is the most effective measure to improve the health of patients with chronic non-communicable diseases such as hypertension, and incorporating the enhancement of self-management ability into the intervention goals of elderly hypertensive patients can delay disease development, prevent complications, and bring long-term clinical benefits.

Self-management is a skill developed by patients during the course of their disease, and is used to manage clinical symptoms, disease treatment, social and psychological changes, and lifestyle improvement (16). Self-efficacy reflects a patient's confidence and ability to achieve a specific goal, and directly affects healthy behavior. The core of the conventional nursing method of elderly hypertensive patients is taking "disease" as the core, monitoring blood pressure, and maintaining timely treatment. In this method, patients mainly rely on medical staff during the treatment process, as they themselves are relatively passive, resulting in poor compliance and difficulties in to exerting self-management enthusiasm and subjective initiative, so that the blood pressure control effect is unsatisfactory. The RAM is an effective guide for nurses in the management of various diseases and is patient centered (17). It holds that humans are an overall adaptive system and an organic whole with biological, psychological, and social attributes continuously interacting with the physical and social environment and using adaptive strategies to continuously adapt to various stimuli in the internal and external environments. The method is presented in four adaptive modes, namely physiological function, self-concept, role function, and interdependence to promote the physical, psychological, and social integrity of patients, and improve health $(18,19)$. This study followed the basic steps of the
RAM, and saw the primary and secondary assessments, nursing problems, and related factors determined for each patient, resulting in individualized and targeted nursing interventions and health education. The results were evaluated to track the intervention effect, reduce ineffective reactions, improve patients' physiological function and role adaptation function, correct wrong self-concepts, reduce dependence levels, improve patients' self-confidence and initiative in participating in disease management, improve their quality of life, and continuously manage problems existing in elderly hypertensive patients (20). The results of this study showed that after the intervention, the scores of self-efficacy, self-management behavior, and the total score of observation group patients were higher than patients in the control group $(\mathrm{P}<0.05)$, indicating that the implementation of RAM based on routine nursing has more advantages, which is consistent with the results of related research (21). According to the analysis, this may be because the RAM requires nurses to communicate with patients in a "one-to-one" manner, strengthening the systematic and comprehensive evaluation of their patients' physiology, psychology, and interactions society, and discovering existing or potential nursing problems not determined using other nursing methods.. Moreover, the model can dynamically assess the most prominent problems faced by patients at different stages and implement targeted interventions so that hurdles to their progress can be resolved in time. This helps elderly hypertensive patients to actively recognize the disease and participate in selfmanagement through the whole process, teaches patients the skills of self-management using diet, medication, mood, exercise, work, and illness monitoring, improves selfmanagement confidence, actively corrects patients' poor mental state, and feeds back blood pressure monitoring results, so that patients can perceive the benefits of selfmanagement.

Hypertension patients must take long-term antihypertensive drugs and medication compliance is the most critical link in determining whether medical treatment will be effective (22). Poor medication compliance can reduce treatment expectations, which leads to poor blood pressure control and a reduced quality of life. Nursing based on the RAM can effectively improve patients' awareness of hypertension prevention and treatment knowledge, enhance their awareness of standardized medications as well as their understanding of the importance of taking medication, and improve compliance. The results of this study showed that after intervention, the observation 
groups medication compliance score was significantly higher than the control group patients $(\mathrm{P}<0.01)$, and the observation groups systolic and diastolic blood pressure were significantly lower than the control group $(\mathrm{P}<0.05)$. The observation groups blood pressure control compliance rate $(85.4 \%)$ was also significantly higher than the control groups $(64.6 \%)(\mathrm{P}<0.05)$, and their physiological function, physiological competency, physical pain, energy, social function, emotional function, mental health scores, and total score in the quality-of-life scale were all higher than the control group $(\mathrm{P}<0.05)$. Moreover, the effect was better than conventional nursing methods, which is in line with the results of previous studies $(23,24)$. However, the comparison of scores between the groups in the general health status dimension was not statistically significant $(\mathrm{P}>0.05)$ and may require further research.

\section{Limitations}

Due to human, financial, and energy constraints, this study has shortcomings. On the one hand, the research object was limited to elderly hypertensive patients treated in our hospital between June 2020 and March 2021. The limitation of sampling range, sample size, and individual differences of patients limit the universality of the study results to other regions and groups, and further experimental studies are needed to observe the effect of intervention and strengthen the verification of the conclusions. In addition, due to time constraints, this study only evaluated the effect of intervention for 3 months, which is relatively short, and without continuous follow-up investigation, it is impossible to judge the long-term effect of disease adaptation intervention based on the RAM. In addition, in the effect evaluation stage after 3 months of intervention, part of the data was collected through telephone follow-up due to difficulties in the face-to-face evaluation of participants.

\section{Conclusions}

In summary, this study evaluated nursing care based on the RAM in the management of routine nursing for elderly hypertension patients. The results showed that after 3 months of intervention, observation group patients had more positive and significant effects in self-efficacy, selfmanagement ability, medication compliance, quality of life, and blood pressure control than control group patients. This shows that nursing methods based on the RAM can be applied to elderly hypertensive patients. These results are of significance for health professionals engaged in hypertension management in choosing the care path for patients. Based on the results of this study, we recommend intervention measures based on the RAM be adopted, that dynamic primary and secondary assessments of patients be conducted, that nursing problems be analyzed, and that health promotion measures be implemented accordingly, so as to further ensure the effectiveness of intervention methods for individual patients. Nursing intervention based on the RAM is conducive to integrating the adaptation process, providing elderly hypertensive patients with coping strategies and self-care skills, and enhancing their self-management behavior and self-management ability. It has a positive effect on promoting healthy behavior change, improving quality of life, improving medication compliance, and achieving better blood pressure control effect, and is worthy of promotion and application.

\section{Acknowledgments}

The authors would like to thank patients and staff from participating departments.

Funding: Funding for this study was provided by the inhospital Nursing Research Fund of the Fifth Affiliated Hospital of Sun Yat-sen University (2019hlky08).

\section{Footnote}

Reporting Checklist: The authors have completed the CONSORT reporting checklist. Available at https://dx.doi. org/10.21037/apm-21-2803

Trial Protocol: Available at https://dx.doi.org/10.21037/apm21-2803

Data Sharing Statement: Available at https://dx.doi. org/10.21037/apm-21-2803

Conflicts of Interest: All authors have completed the ICMJE uniform disclosure form (available at https://dx.doi. org/10.21037/apm-21-2803). The authors have no conflicts of interest to declare.

Ethical Statement: The authors are accountable for all aspects of the work in ensuring that questions related to the accuracy or integrity of any part of the work are appropriately investigated and resolved. All procedures 
performed in this study involving human participants were in accordance with the Declaration of Helsinki (as revised in 2013). The study was approved by Ethics Committee of the Fifth Affiliated Hospital of Sun Yat-sen University (No. k242-1) and informed consent was taken from all the patients.

Open Access Statement: This is an Open Access article distributed in accordance with the Creative Commons Attribution-NonCommercial-NoDerivs 4.0 International License (CC BY-NC-ND 4.0), which permits the noncommercial replication and distribution of the article with the strict proviso that no changes or edits are made and the original work is properly cited (including links to both the formal publication through the relevant DOI and the license). See: https://creativecommons.org/licenses/by-nc-nd/4.0/.

\section{References}

1. Wang J, Zhang L, Wang F, et al. Prevalence, awareness, treatment, and control of hypertension in China: results from a national survey. Am J Hypertens 2014;27:1355-61.

2. WHO. Hypertension. World Health Organisation. 2020. Available online: https://www.who.int/news-room/factsheets/detail/hypertension, accessed 26 Feb 2020.

3. Gillespie CD, Hurvitz KA. Centers for Disease Control and Prevention (CDC). Prevalence of hypertension and controlled hypertension - United States, 2007-2010. MMWR Suppl 2013;62:144-8.

4. Verdecchia P, Reboldi G, Angeli F. The 2020 International Society of Hypertension global hypertension practice guidelines - key messages and clinical considerations. Eur J Intern Med 2020;82:1-6.

5. Perrett SE. Review of Roy adaptation model-based qualitative research. Nurs Sci Q 2007;20:349-56.

6. Roy C. Extending the Roy adaptation model to meet changing global needs. Nurs Sci Q 2011;24:345-51.

7. de Oliveira TC, de Araújo TL, Melo EM, et al. Evaluation of the adaptation process in elderly patients with arterial hypertension. Rev Lat Am Enfermagem 2002;10:530-6.

8. Jennings KM. The Roy Adaptation Model: A Theoretical Framework for Nurses Providing Care to Individuals With Anorexia Nervosa. ANS Adv Nurs Sci 2017;40:370-83.

9. Unger T, Borghi C, Charchar F, et al. 2020 International Society of Hypertension global hypertension practice guidelines. J Hypertens 2020;38:982-1004.

10. Yang B, Li X. The effect of nursing intervention on selfefficacy of hospitalized patients with hypertension. Int J
Nurs Sci 2011;30:1377-8.

11. Zhao QL. Reliability and validity of the Hypertension Patients Self-Management Behavior Rating Scale (HPSMBRS). Chin Nurs Management 2012;12:26-31.

12. Morisky DE, Ang A, Krousel-Wood M, et al. Predictive validity of a medication adherence measure in an outpatient setting. J Clin Hypertens (Greenwich) 2008;10:348-54.

13. Alkan H, Yildiz N, Ardiç F. The Correlations Between Disease Specific Quality of Life, Short Form-36 and Clinical Variables in Patients With Ankylosing Spondylitis. Arch Rheumatol 2020;35:468-76.

14. Bao M, Wang L. The longitudinal trend of hypertension prevalence in Chinese adults from 1959 to 2018: a systematic review and meta-analysis. Ann Palliat Med 2020;9:2485-97.

15. Zhang Q, Huang F, Zhang L, et al. The effect of high blood pressure-health literacy, self-management behavior, self-efficacy and social support on the health-related quality of life of Kazakh hypertension patients in a lowincome rural area of China: a structural equation model. BMC Public Health 2021;21:1114.

16. Eck C, Biola H, Hayes T, et al. Efficacy of Hypertension Self-Management Classes Among Patients at a Federally Qualified Health Center. Prev Chronic Dis 2021;18:E70.

17. Fawcett J, Desanto-Madeya S. Contemporary nursing knowledge: analysis and evaluation of nursing models and theories. Philadelphia: FA Davis Company, 2013.

18. Parker MM, Smith MC. Nursing theories and nursing practice. Philadelphia: FA Davis Company, 2010.

19. Shariatpanahi S, Ashghali Farahani M, Rafii F, et al. Designing and Testing a Treatment Adherence Model Based on the Roy Adaptation Model in Patients With Heart Failure: Protocol for a Mixed Methods Study. JMIR Res Protoc 2019;8:e13317.

20. Amanak K, Sevil U, Karacam Z. The impact of prenatal education based on the Roy adaptation model on gestational hypertension, adaptation to pregnancy and pregnancy outcomes. J Pak Med Assoc 2019;69:11-7.

21. Wang X, Tang L, Howell D, et al. Theory-guided interventions for Chinese patients to adapt to heart failure: A quasi-experimental study. Int J Nurs Sci 2020;7:391-400.

22. Hosseininasab M, Jahangard-Rafsanjani Z, Mohagheghi A, et al. Self-monitoring of blood pressure for improving adherence to antihypertensive medicines and blood pressure control: a randomized controlled trial. Am J Hypertens 2014;27:1339-45.

23. Bockwoldt D, Staffileno BA, Coke L, et al. Understanding Experiences of Diabetes Medications Among African 
Americans Living With Type 2 Diabetes. J Transcult Nurs 2017;28:363-71.

24. Turan Kavradim S, Canli Özer Z. The effect of education and telephone follow-up intervention based on the Roy Adaptation Model after myocardial infarction: randomised controlled trial. Scand J Caring Sci 2020;34:247-60.

Cite this article as: Zhang J, Guo L, Mao J, Qi X, Chen L, Huang H, Sun Y, Yang X. The effects of nursing of Roy adaptation model on the elderly hypertensive: a randomised control study. Ann Palliat Med 2021;10(12):12149-12158. doi: 10.21037/apm-21-2803 